\title{
NTM induced fast ion losses in ASDEX Upgrade
}

\author{
M. García-Muñoz ${ }^{(1)}{ }^{*}$ P. Martin ${ }^{(2,3)}$, H.-U. Fahrbach ${ }^{(1)}$, M. Gobbin ${ }^{(2,3)}$, S. Günter ${ }^{(1)}$, \\ M. Maraschek ${ }^{(1)}$, L. Marrelli ${ }^{(2)}$, H. Zohm ${ }^{(1)}$, and the ASDEX Upgrade Team \\ (1) Max-Planck-Institut für Plasmaphysik, EURATOM Association \\ Boltzmannstr. 2, D-85748 Garching, Germany \\ ${ }^{(2)}$ Consorzio RFX, Associazione EURATOM-ENEA per la fusione, Padova, Italy and \\ ${ }^{(3)}$ Physics Department, University of Padova, Italy
}

(Dated: March 27, 2008)

\begin{abstract}
The loss of fast (i.e. suprathermal) ions from a magnetically confined fusion plasma due to the interaction with magnetohydrodynamic instabilities has been experimentally characterized and interpreted by means of a numerical model. It is found that for a special class of instabilities, the so-called Neoclassical Tearing Modes, fast ions losses are increased and modulated at the same frequency of the mode. This new experimental finding is explained as a result of the drift islands formed by energetic ions in particle phase space. An eventual overlapping of these drift islands leads to an orbit stochasticity and therefore to an enhancement of the fast ion losses. This explanation is confirmed by statistical analysis of simulations of fast ions trajectories performed with the ORBIT code. The mechanism is of general importance for understanding the interaction between MHD modes and fast particles in magnetic confinement experiments.
\end{abstract}

PACS numbers: 52.35.Fi, 52.35.Py, 52.50.Gj, 52.55.Fq, 52.55.Pi

A significant fraction of plasma pressure in a magnetized fusion experiment is carried by suprathermal (fast) ions, which are produced either by fusion reactions (like $\alpha$ particles), injected through energetic beams, or by RF heating. In general, these fast particles play an important role in the energy balance of a fusion plasma, either for the heating and/or for current drive processes [1], [2] and [3]. The confinement of fast particles is therefore an issue of great importance, since significant losses of these ions may drastically reduce the heating as well as the current drive efficiency. In addition, an intense and localized loss of fast ions may cause damage to plasma facing components. Due to their high energy, the dynamics of fast ions in a magnetized plasma is rather different than that of thermal ions and, in many aspects, still experimentally unexplored. Several issues are still open, for example, about the interplay between a population of fast particles and a key player of magnetized fusion plasmas, like the Magnetohydrodynamic (MHD) instabilities.

In this Letter we present the first measurements of fast ion losses with simultaneous high time, energy and, pitch angle resolution due to Neoclassical Tearing Modes (NTMs). We explain the measurements on the basis of drift islands and their overlap, which leads to orbit stochasticity. The main experimental phenomenology is in fact reproduced by a model simulating the guiding center orbits of fast ions. The results reported here are important for next-step fusion devices like ITER where a significant population of $\alpha$-particles and of NBI and ICRH generated fast ions will be present.

NTMs are metastable modes driven by the missing bootstrap current within a preexisting seed magnetic is-

\footnotetext{
*Electronic address: Manuel.Garcia-Munoz@ipp.mpg.de
}

land, provided that the plasma poloidal beta, $\beta_{p o l}$, is larger than a threshold value [4]. When a NTM grows in the plasma, global confinement is severely affected. NTMs set the limit to the maximum $\beta_{\text {pol }}$ achievable in conventional scenarios. While the NTM impact on the global confinement is rather well established, less is known on how they influence energetic particles, like for example ICRH heated ions, or ions of NBI (Neutral Beam Injection) origin. Experiments on this subject have been performed in TFTR [5], [6] and DIII-D [7]. The study of the effect of NTMs on the fast ion confinement is important, for example, to fully asses the efficiency of NBI heating and current drive. In next generation devices, like ITER, this information is important also to predict the confinement of alpha particles and the impact of their losses on plasma facing components.

The ASDEX Upgrade (AUG) tokamak [8] has unique capabilities for fast particle studies: its heating system, with $20 \mathrm{MW}$ of NBI at $60 / 93 \mathrm{keV}, 6 \mathrm{MW}$ of ICRH and 2 MW of ECRH, allows a rich variety of scenarios, where the behavior of fast ion population can be finely tuned and decoupled from the bulk plasma environment. AUG is equipped with 8 NBI sources, each one capable of supplying 2.5 MW at different injection angles.

A new diagnostic in AUG, the fast ion loss detector (FILD), provides energy and pitch-angle resolved measurements of fast ion losses, with a bandwidth of $1 \mathrm{MHz}$ [9]. Its design is based on the concept of the $\alpha$-particle detector used for the first time at TFTR [10] and more recently at W7-AS [11]. The active part of the diagnostic is a scintillator plate contained within a cylindrical cup, which can be inserted via a movable manipulator up to a few $\mathrm{mm}$ behind the limiter slightly above the mid plane. Fast ions enter the detector through an aperture open in the cup and hit the scintillator. Their strike points depend on fast particles gyroradius (i.e. their energy) 
and on their pitch angle, defined as the angle between their velocity and the local magnetic field B, Fig.1. The scintillating surface is observed via a CCD camera, which provides a slow but highly spatially resolved image, and by an array of 20 photomultipliers, which have a bandwidth of $1 \mathrm{MHz}$ and provide therefore a very high time resolution.
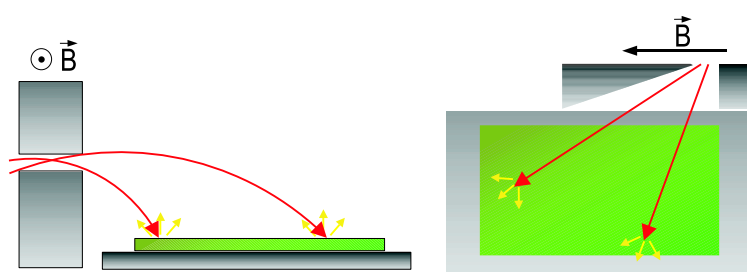

FIG. 1: Schematic of the fast ion loss detector principle. The probe consists basically of one aperture which disperses the NBI ion losses according to gyroradius and pitch angles onto the scintillator. Two different particle trajectories have been drawn, showing how the aperture works in the perpendicular and parallel direction to the magnetic field.

The experiments discussed in this Letter have been mainly performed in plasmas with toroidal current $I p=$ $0.8 M A$, toroidal field $B_{t}=2 T$, safety factor at the edge $q_{95}=4.5$ and NBI as main heating and fast particle source. Time traces of the total NBI power and the magnetic fluctuations due to the $(m=2, n=1)$ and the $(\mathrm{m}=3, \mathrm{n}=2)$ NTM for a typical discharge $(\# 21089)$ are shown in the bottom of Fig.2. An NBI power ramp in the period $[1-2]$ s excites a $(2,1)$ NTM, which changes strongly in frequency and resonance location during the NBI modulation phase $[2-3] \mathrm{s}$. Between $t=3 \mathrm{~s}$ and $t=5 \mathrm{~s}$ the mode reaches a plateau phase in which it maintains constant its main features i.e. frequency, resonance location and amplitude. This phase has been selected to study the properties of the $(2,1)$ NTM induced fast ion loss and its dependence on the mode amplitude.

In order to identify the lost particles in phase space we analyze the loss pattern recorded by the CCD camera during the MHD activity. On top, Fig.2 shows a CCD frame for the discharge \#21089 at $t=4.03 \mathrm{~s}$, when the NTM is present; $(2,1)$ NTM induced fast ion losses together with the prompt losses generated by three different NBI sources are visible. The more radially injected ions (sources \#3 and \#5) appear in the region of higher pitch angle $\left(70^{\circ}-75^{\circ}\right)$ while a tangential source (\#7) produces prompt losses in a lower pitch angle region $\left(50^{\circ}-60^{\circ}\right)$. The pitch angle is defined as $\arccos \left(\frac{v_{\|}}{v_{t o t}}\right)$. The $(2,1)$ NTM contribution to the fast ion loss pattern shows a pitch angle selective character with two main components. First, a loss of fast ions appears only when the $(2,1)$ NTM is present in the phase space region corresponding to low pitch angles $\left(35^{\circ}-45^{\circ}\right)$ and to the NBI main injection energy, as reported in Fig.2. In addition, the prompt loss pattern due to the NBI source \#5 and \#7 shows (when a $(2,1)$ NTM is present) a broader energy distribution which ranges from the injection energy, 93
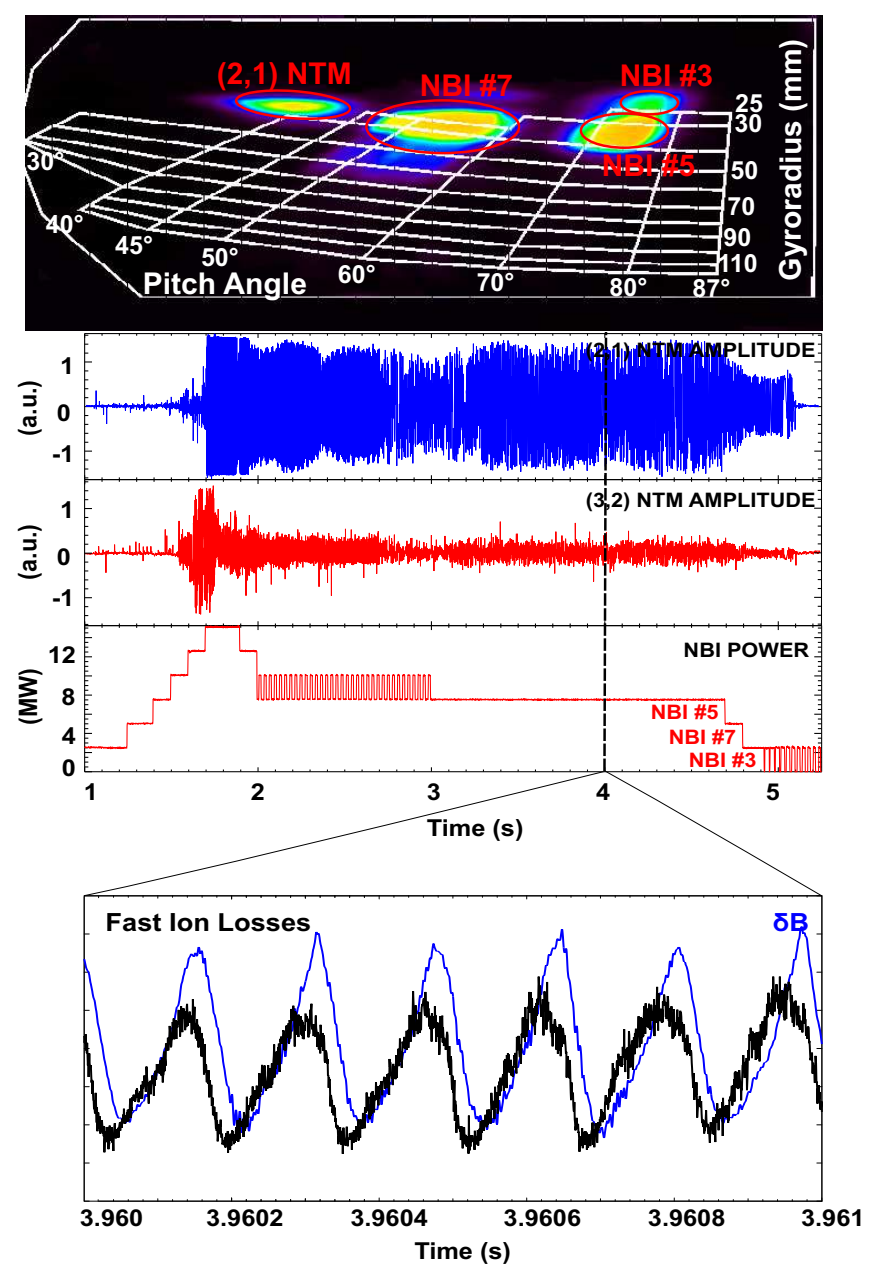

FIG. 2: AUG discharge \#21089: CCD view showing the three spots produced by first orbit losses from NBI sources \#3, \#5 and \#7 and $(2,1)$ NTM induced fast ion losses. On the bottom, the magnetic activity is shown by the Odd-N and Even- $\mathrm{N}$ magnetic pick-up coil signals together the total NBI power. The lock-phase between the $(2,1)$ magnetic perturbation amplitude $\delta \mathrm{B}(\mathrm{t})$ and the fast ion losses is also shown.

$\mathrm{keV}$ (gyroradius of $40 \mathrm{~mm}$ ) down to the minimal measurable energy, $60 \mathrm{keV}$ (gyroradius of $25 \mathrm{~mm}$ ). Fig.2 shows as well the magnetic activity present in the discharge by means of the N-Odd and N-Even magnetic signals together with the total NBI power. On the bottom, Fig.2 shows the main finding of this work, the lock-phasing between the $(2,1)$ NTM magnetic perturbation and the fast ion losses (FIL) during a time window of $10 \mathrm{~ms}$. During this phase the experimental magnetic fluctuation level at the wall was $B_{r}(t) \approx 40 m T$.

To quantify the amount of lost particles due to the NTM magnetic perturbation and to separate those from the common NBI prompt losses, a Fast Fourier Transformation (FFT) was applied to the signal of the photomultipliers which cover the phase space regions where NTM induced losses are observed, Fig.3-a. The same FFT was 

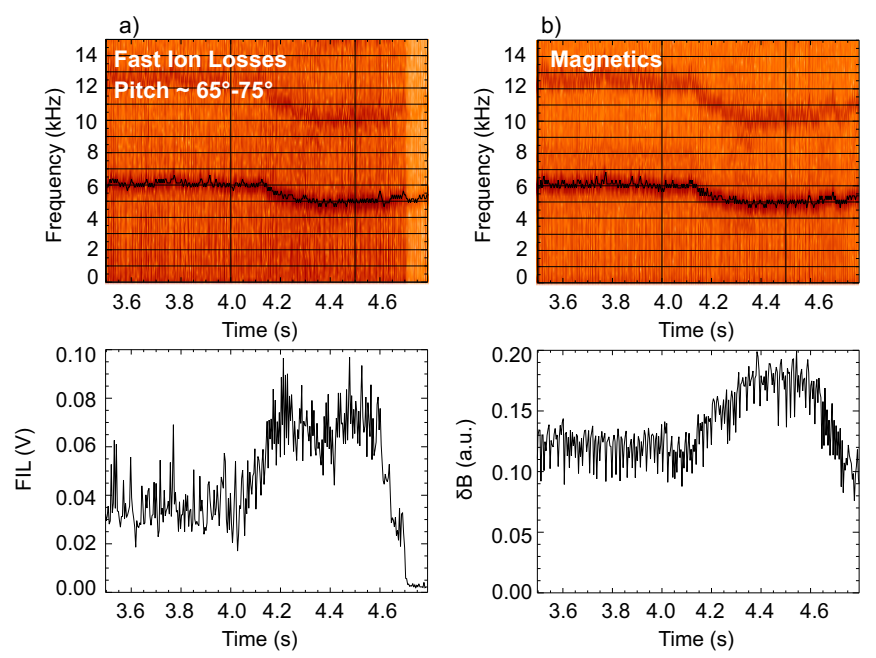

FIG. 3: AUG discharge \#21089: Amplitude analysis of the $(2,1)$ NTM magnetic perturbation $\delta \mathrm{B}(\mathrm{t})$ and their corresponding fast ion losses at a pitch angle $\approx 65^{\circ}-75^{\circ}$ and gyroradii of $\approx 40 \mathrm{~mm}$.

also applied to the magnetic perturbation recorded by the Mirnov coils, Fig.3-b. We observe a correlation between the frequency and phase of the mode and those of the losses. Tracking the main frequency of magnetic perturbation and of the fast ion losses we find, in general, a strong correlation between the NTM amplitude and the energetic particle loss signal.

The intermittent operation of NBI sources, with a switch-off time shorter than $50 \mu s$, has been used to provide a periodically changing source of fast particles which helps to study the loss time scales. In discharge \#21168, the NBI source \#8 (which is injecting rather radially deuterium ions at $93 \mathrm{keV}$ ) has been modulated with square pulses of 2.5 MW amplitude, on top of a constant background of $5 \mathrm{MW}$. In this discharge, a large $(2,1)$ magnetic island is the responsible for the fast ion losses. A detailed analysis of the loss time scales reveals a different behavior depending on the pitch angles of the lost ions. The losses of deuterons with energies $E \approx 93 \mathrm{keV}$ and rather parallel velocities (i.e. pitch angles $\approx 35^{\circ}-45^{\circ}$ ), injected by the modulated beam, closely follow both the time evolution of their source and the mode evolution. Fig.4-a shows the spectrogram of the FILD channel \#7 corresponding to $E \approx 93 \mathrm{keV}$ and pitch angle $\approx 35^{\circ}-45^{\circ}$. Besides the slow trend following the frequency evolution of the mode and of its harmonics, we note discrete spots corresponding to the NBI modulation. As shown in bottom of Fig.4-a, the amplitude of the losses at the dominant mode frequency (black curve) is modulated according to the NBI evolution (red curve) and its envelope follows the amplitude of the magnetic mode (blue curve). Looking more closely into an individual NBI square pulse, Fig.4-b, we observe that the losses recorded by the FILD channel \#7 promptly decay as soon as the modulated source is switched-off, thus indicating a time scale for these losses not larger than a few tens of $\mu s$. The FILD channel \#9, which corresponds to lost particles with $E \approx 93 \mathrm{keV}$ and pitch angles $\approx 70^{\circ}-75^{\circ}$, shows different features. While the modulation pattern is still recognizable, after the beam switch-off there is a tail in the loss signal, corresponding to a time scale of the order of few ms for this kind of particles. Both signals show a strong correlation between the amount of fast ion losses and the amplitude of the magnetic perturbation.

The absolute amount of lost ions has been estimated taking into account the photomultiplier signals and the number of photons produced by each incident ion in the scintillator plate (ionoluminiscense). The scintillator ionoluminescense was calibrated in an accelerator facility [9]. The typical lost ion flux varies from $4 \cdot 10^{13} \frac{\mathrm{Ions}}{\mathrm{s} \cdot \mathrm{cm}^{2}}$ for the NTM prompt losses up to $10^{14} \frac{\mathrm{Ions}}{\mathrm{s} \cdot \mathrm{cm}^{2}}$ for the lost ions with higher pitch angle. Both signals are of the same order as the maximal NBI prompt loss signal, $4 \cdot 10^{14} \frac{\text { Ions }}{\mathrm{s} \cdot \mathrm{cm}^{2}}$. Comparing the NTM induced fast ion losses with the NBI prompt losses and taking into account that the first ones should be toroidally distributed and the prompt losses are well localized, it makes clear the importance of this study.

The experimental findings presented in this Letter are discussed in the framework of a mechanism, which involves drift islands in the fast particle orbit space [12].

This mechanism is the result of the interaction of energetic ion motion with a magnetic equilibrium perturbed by a long-wavelength mode. A single helicity magnetic perturbation, like that due to the $(2,1)$ NTM, produces an island chain in the magnetic field. These magnetic islands do not cause significant ergodicity of magnetic field lines (followed by the guiding centers of thermal particles) for the amplitudes observed here. The situation for fast particles is different: the coupling between the fast particles guiding center motion in the perturbed magnetic field and the orbit shift due to the drifts (which has a $(m=1, n=0)$ character) results in several chains of drift islands (of $(1,1),(2,1),(3,1)$ and $(4,1)$ helicities) in fast particle phase space. Depending on the shape of the $q$-profile, on the location of the $q=2$ resonance and on the amplitude of the original $(2,1)$ mode, these islands may or may not overlap, but in both cases they may drive fast particle losses. If there is overlapping, a stochastic region is created in the fast particles orbit space, which originates significant losses [12].

This problem has been studied by computing the trajectories of fast ions, injected by NBI in a tokamak magnetic equilibrium perturbed by a $(2,1)$ mode. The simulation has been performed with the Hamiltonian guiding center code ORBIT [13], which is that used in [5] and [7]. For this study a circular cross section equilibrium has been considered: this choice strongly facilitates the problem from a numerical point of view, with the drawback that the comparison with the experiment can not be pushed up to very quantitative detail.

The initial fast ion population has been chosen as that resulting by the ionization pattern of the applied NBI 
a)

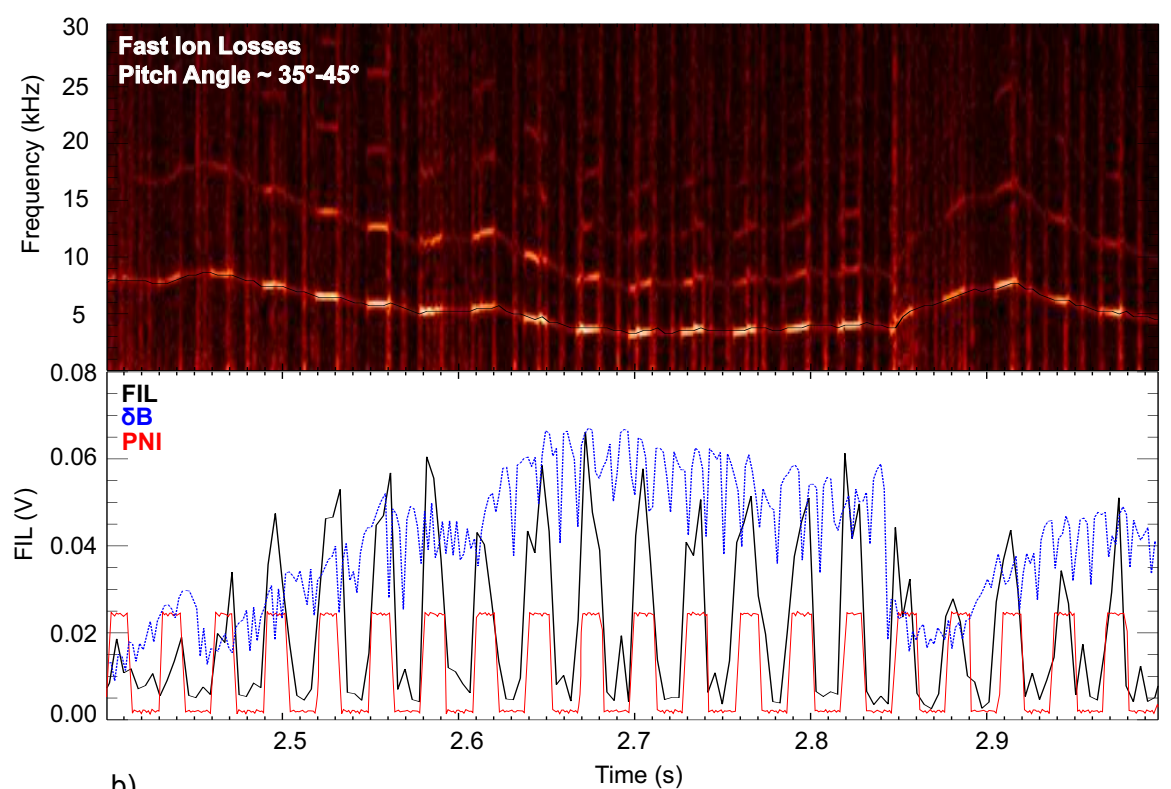

b)
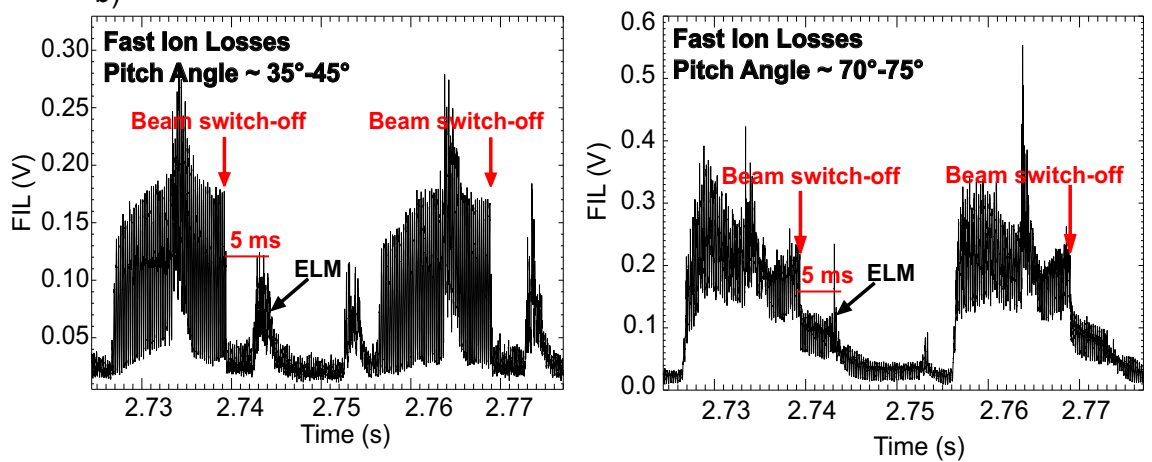

FIG. 4: AUG discharge \#21168: a) Amplitude analysis of fast ion losses during the $(2,1)$ magnetic island activity. The blue line depicts the amplitude of the magnetic perturbation. The NBI modulation is drawn by a red line. b) Two FILD channels are presented, i.e. two pitch angle regions, showing different temporal behaviors.

sources in a plasma with global parameters corresponding to those of the plasma in Fig.3. The ionization pattern was calculated using the FAFNER code [14], which provides the birth location, energy and pitch angle of the injected NBI ions. The pitch angle of the ions at the starting point depends on the injection direction of the NBI source and the local magnetic field. The resulting distribution of about 90000 ions with energies above 90 $\mathrm{keV}$, originally produced for the D-shaped plasma, has been re-mapped into the circular equilibrium and was taken as the initial particle distribution for ORBIT. A circular cross-section tokamak equilibrium with the same AUG major radius, and minor radius equal to the average between the two semi-axis of the AUG D-shaped cross section, a toroidal field $B_{t}=2 T$ and a superimposed stationary $(\mathrm{m}=2, \mathrm{n}=1)$ perturbation, has been considered. The perturbation for the $(2,1)$ NTM implemented in the code is stationary and is given by $\delta \mathbf{b}=\nabla \times \alpha \mathbf{B}_{\mathbf{0}}$ with the following expression for the eigenfunction $\alpha$ :

$$
\alpha\left(\Psi_{p}, \zeta, \theta\right)=\alpha_{0} r\left(\psi_{p}\right)^{m}\left(\psi_{p}-\psi_{w}\right) \sin (n \zeta-m \theta+\Phi)
$$

where $(m, n)$ is the helicity of the mode, $r\left(\psi_{p}\right)$ is the normalized radius as function of the poloidal flux, $\psi_{w}$ is the poloidal flux at the wall, and $\Phi$ is the mode phase.

Its amplitude has been chosen to produce a magnetic island size consistent with experimental measurements, i.e. $\approx 10-12 \mathrm{~cm}$ in the low field side. Interactions of fast ions with the background plasma, i.e. pitch angle scattering and slowing down have been included in the simulations. The trajectories of a set of test fast particles all starting at $\mathrm{t}=0$ are integrated over time and all informations about particles that reach the wall are recorded. The first evidence is that the presence of the mode causes a significant enhancement of the fraction of simulated fast particles reaching the wall, mostly concentrated in a toroidally localized region (which, as we will see in Fig.6, it is a signature of the $n=1$ character of the losses) and close to the equatorial mid plane. With the mode eigenfunction used in this paper, an amount of losses of the order of $10 \%$ of the injected fast ion population is observed within a few ms. This number is 


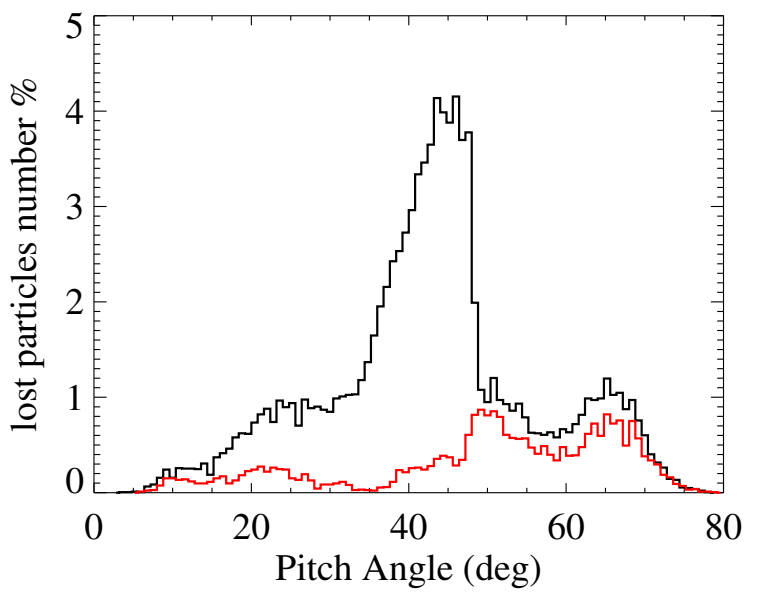

FIG. 5: Histograms of the pitch angles of particles lost during the ORBIT simulation with perturbation (black line) and without it (red line).

only indicative, since the assumptions used in the model, and in particular the circular geometry instead than the D-shaped geometry, do not allow a precise quantitative estimate of the losses. The elongation of the flux surfaces could for example reduce the drift orbit displacement.

In Fig.5 we show the distribution of the pitch of lost ions. For this figure, we have considered the particles lost within a limited region in toroidal and poloidal angles, where the effect of the mode is concentrated, see Fig.6. All these particles have basically their birth energy, i.e. $93 \mathrm{keV}$. For comparison the distribution of the losses without the mode is also shown (red curve); the two peaks in the distribution without the mode correspond to first orbit losses for the two $93 \mathrm{keV}$ beams used in this simulation. The increase of losses is different for particles of different pitch: in particular a large increase is observed around $45^{\circ}$. This peak has interesting analogies with the experimental observations. At this pitch angle, in fact, a spot is found in the CCD image (that labeled (2,1) NTM in Fig.2). The experimental losses corresponding to that spot appear only when the mode is present, a fact, which is consistent with the prediction of the appearance of the peak in Fig.5. A peak in the numerical losses is observed also around $70^{\circ}$, which is consistent with the corresponding experimental spot due to NBI source \#3 (see Fig.2). The increase of losses in the simulation due to the mode is much smaller than for the pitch region around $45^{\circ}$. This might be due to the fact that these particles are trapped, and in the numerical simulation magnetic ripple and mode rotation have not been included. The peak around $50^{\circ}-55^{\circ}$ corresponding to the NBI source \#7 is not evident in the simulation, though losses slightly increase in that region. Likely we have a merging with the adjacent peak, possibly due to the limits of the model. Moreover, for com-
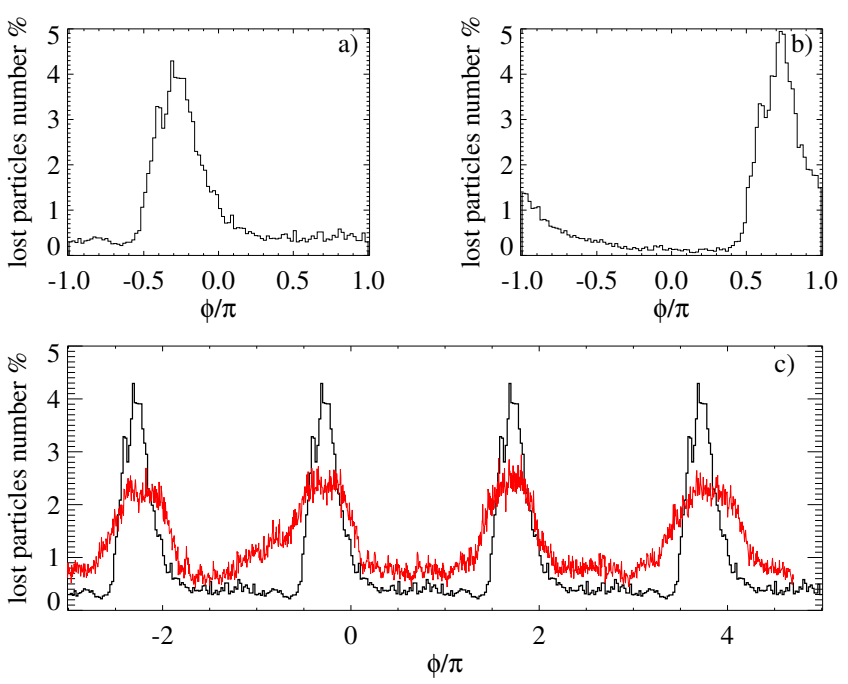

FIG. 6: Toroidal angle distribution of simulated losses as a function of the toroidal angle, $\phi$. The two panels a) and b) show histograms for two choices of the phases of the $(\mathrm{m}=2, \mathrm{n}=1)$ mode, differing by $\pi$. In $\mathrm{c})$ a comparison between the simulation (black line) and the experimental data (red line) is shown.

putational reasons, statistically significant results are obtained integrating losses over a range of toroidal angle, while the measurement is toroidally localized. It is worth noting that the simulation also shows losses at pitch angles $<35^{\circ}$, which can not be detected by the FILD.

In order to compare the simulation results with the FILD time traces, the distribution of the toroidal loss angles for particles with a pitch $\approx 45^{\circ}$ (corresponding to the peak of losses in presence of the NTM) is shown in Fig.6-a: it is characterized by a single peak, reflecting the $n=1$ nature of the losses. The location of the peak is correlated to the phase of the magnetic perturbation: Fig.6-b shows the histogram of the losses for a simulation where the phase of the $(m=2, n=1)$ perturbation was displaced by $\pi$. This is in agreement with the experimentally observed modulation of the losses at the frequency of the NTM (Fig.3). Moreover, a direct comparison with experimental data can be done by reporting them on the toroidal distribution of the simulated losses. The result is shown in Fig.6-c where a few periods of the FILD signal have been superimposed to the simulated toroidal distribution (replicated several times) by mapping time into toroidal angle, assuming a rotation with constant angular velocity and adjusting the initial phase to match the maximum. The amplitude of the signal is multiplied only by a constant without any change in the offset. The agreement with the experiment is qualitatively good. Finally we considered the distribution of the times at which simulated particles were lost. The integral probability distribution of loss times for the lost particles described in 


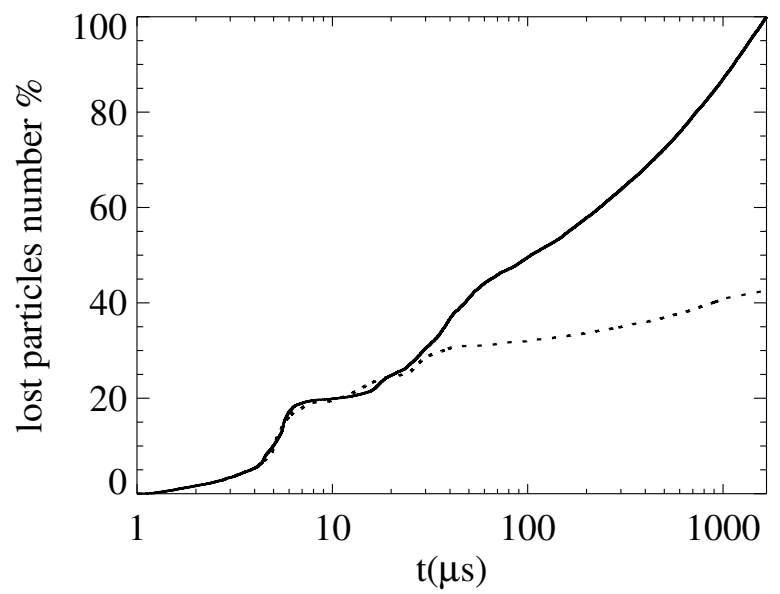

FIG. 7: Integral probability distribution of the loss times of fast particles with energy $E>90 \mathrm{keV}$ in the presence of the perturbation (solid line) and without it (dashed line). Both curves are normalized to the number of $E>90 \mathrm{keV}$ particles lost in the simulation with an NTM.

Fig. 5 is reported in Fig.7, for the case with NTM (solid curve) and without (dashed curve). For a given value in the $\mathrm{x}$-axis, the curve provides the number of lost particles, which leave the plasma within that time. Particles with energy close to their birth value (93 keV), which are those experimentally recorded, are typically lost within that time range. The plot shows that particles are lost in a broad range of time scales: while the first are promptly lost after $\approx 10 \mu \mathrm{s}$ (to be compared with the toroidal transit time for $100 \mathrm{keV}$ deuterons equal to $5 \mu \mathrm{s}$ ) a fraction of the population takes a longer time before escaping. This phenomenology is similar to the experimental observation of ions being lost on a slow time scale (Fig.4 lower right frame). In fact, the particles with the shortest loss times are those deposited by the NBI system on the high field side well inside the separatrix, and cross readily on the low field side due to the torus drift displacement of their orbits. The modulation with the island frequency can be explained by the drift islands with $(3,1)$ and $(4,1)$ helicities. Particle losses after longer times (hundreds of $\mu s$ till $m s$ time scale) are those more influenced by the stochastic diffusion. The comparison between the model and the experiment as far as loss times are concerned needs anyway to be considered as qualitative, because of the before mentioned limits of the numerical simulation.

In conclusion, the losses of fast ions driven by Neoclassical Tearing Modes have been investigated in a variety of plasmas produced in ASDEX Upgrade. The experimental data indicate that NTMs and in general magnetic islands produced by slow MHD modes are responsible for an important enhancement of the outwards radial transport of NBI generated fast ions. A mechanism, based on the drift islands in the particle phase space, has been identified as the process responsible for these losses being the numerical predictions consistent with experimental data.

\section{Acknowledgments}

We wish to acknowledge the valuable support of V. Igochine, P. Piovesan, M. Reich, A. Stäbler, E. Strumberger and R. B. White (Princeton Plasma Physics Laboratory, PPPL).
[1] W. W. Heidbrink et al, Nuc. Fusion 34, 535 (1994).

[2] S. E. Sharapov et al, Nuc. Fusion 40, 1363 (2000).

[3] S.-D. Pinches, Plasma Phys. Contr. Fusion 46, B187 (2004).

[4] Z. Chang et al, Phys. Rev. Letter 74, 4663 (1995).

[5] S. J. Zweben et al, Nuc. Fusion 39, 1097 (1999).

[6] S. J. Zweben et al, Nuc. Fusion 40, 91 (2000).

[7] E. M. Carolipio et al, Nuc. Fusion 42, 853 (2002).

[8] A. Herrmann, Fusion Science and Techn. 44, 569 (2003).

[9] M. García-Muñoz, Ph.D. thesis, Fast Response Scintillator Based Detector for MHD Induced Energetic
Ion Losses in ASDEX Upgrade, Ludwig-MaximillianUniversity of Munich (2006).

[10] D. Darrow and Weller, A., Rev. Sci. Instrum. 66, 476 (1995).

[11] A. Werner, A. Weller, Rev. Sci. Instrum. 72, 780 (2001).

[12] H. E. Mynick, Phys. Fluids B 5, 1471 (1993).

[13] R. B. White, Phys. Fluids 27, 2455 (1984).

[14] G. G. Lister, Tech. Rep., Max-Planck-Institut Fuer Plasmaphysik IPP 4/222 (1985). 